MODELING, IDENTIFICATION AND CONTROL, 2001, VOL. 22, NO. 1, 15-27

doi:10.4173/mic.2001.12

\title{
Computational Performance Analysis of Nonlinear Dynamic Systems using Semi-infinite Programming*
}

\author{
TOR A. JOHANSEN $\dagger$
}

Keywords: Hamilton-Jacobi inequality, stability, Lyapunov functions, convex optimization, performance

\begin{abstract}
For nonlinear systems that satisfy certain regularity conditions it is shown that upper and lower bounds on the performance (cost function) can be computed using linear or quadratic programming. The performance conditions derived from Hamilton-Jacobi inequalities are formulated as linear inequalities defined pointwise by discretizing the state-space when assuming a linearly parameterized class of functions representing the candidate performance bounds. Uncertainty with respect to some system parameters can be incorporated by also gridding the parameter set. In addition to performance analysis, the method can also be used to compute Lyapunov functions that guarantees uniform exponential stability.
\end{abstract}

\section{Introduction}

This work describes a procedure for computing upper and lower performance bounds in terms of solutions to Hamilton-Jacobi inequalities, including Lyapunov functions as a special case. This is an interesting problem because it may give information about to what extent a controller meets its performance specifications in terms of a cost function when the system is subject to uncertainty, the control design is suboptimal or based on a simplified model.

Consider the equilibrium point $x=0$ of the non-autonomous non-linear systems

$$
\begin{array}{rlrl}
x(t+1) & =f(x(t), \theta(t)) & & \text { D-T } \\
\dot{x}(t) & =f(x(t), \theta(t)) & \text { C-T }
\end{array}
$$

where $x \in X$ is the state vector, $\theta \in \Theta$ is an unknown possibly time-varying parameter vector, $f(0, \theta)=0$ for all $\theta \in \Theta$ and $f$ is Lipschitz. D-T denotes discrete time, while C-T denotes continuous time. Let $X \subset R^{n}$ be a compact and connected region of the state space such that the origin is an interior point in $X$, and $\Theta \subset R^{d}$ a compact region of the parameter space. The problem is essentially to compute a smooth function $V: X \rightarrow R$ that satisfies some Hamilton-Jacobi inequality in addition to non-negativity. By assuming a linear parameterization of $V$, this leads to two linear inequalities that must hold for every $x \in X$ and $\theta \in \Theta$. By discretizing the compact sets $X$ and $\Theta$, the candidate functions are now characterized (approximately due to the discretization) by a finite number of linear inequalities.

The present work extends (Johansen 2000) in the sense that more general perfor-

\footnotetext{
*This work was sponsored by the European Commission under the ESPRIT Long Term Research project $28104 \mathscr{H}^{2} \mathscr{C}$.

†Department of Engineering Cybernetics, Norwegian University of Science and Technology, N-7491 Trondheim, Norway. Email: Tor.Arne.Johansen@itk.ntnu.no.

(C) 2000 IEEE. Reprinted, with permission, from Proceedings of the 39th Conference on Decision and Control, Sydney, Australia, December 2000.
} 
mance measures are considered, not just Lyapunov stability. Compared to Rantzer (1999) who represent the performance measure through pointwise function values, a smooth parameterization of $V$ is assumed. Further differences lies in the analysis of the discretization, and the procedure in the present paper allows somewhat more flexibility in specifying the objective through a convex quadratic cost funciton. In Johansson and Rantzer (1998), Rantzer and Johansson (2000), Petterson and Lennartson (1997) and Johansen (1999), who mainly consider performance and Lyapunov function computations for various classes of nonlinear systems the solution is computed using semi-definite programming. Piecewise linear Lyapunov function are computed using linear programming in Ohta and Onishi (1999) and Julian et al. (1999), and solutions to Hamilton-Jacobi-Bellman inequalities for hybrid systems using linear programming are investigated in Branicky (1995) and Hedlund and Rantzer (1999).

\section{Parameterization of the performance bound}

In this section a linearly parameterized set of functions is introduced. Consider a function $V: X \rightarrow R$ of the form

$$
V(x)=x^{T} P(x) x
$$

where the matrix valued function $P: X \rightarrow R^{n \times n}$ is defined by the following linear parameterization

$$
P(x)=\sum_{i=1}^{N} P_{i} \rho_{i}(x)
$$

where $\rho_{i}: X \rightarrow R$ are smooth basis-functions for all $i=1,2, \ldots, N$ and $P_{1}, P_{2}, \ldots, P_{N}$ are parameter matrices. The attention is restricted to positive semi-definite basisfunctions that form a partition-of-unity:

$$
\sum_{i=1}^{N} \rho_{i}(x)=1, \quad \text { for all } x \in X
$$

The set of functions $V$ defined by (3)-(5) and fixed basis-functions $\rho_{1}, \rho_{2}, \ldots, \rho_{N}$ is denoted

$$
\mathscr{V}_{N}=\left\{x \mapsto \sum_{i=1}^{N} x^{T} P_{i} x \rho_{i}(x) \mid P_{1}, P_{2}, \ldots, P_{N} \in R^{n \times n}\right\}
$$

In the procedure developed below, we will make no further assumptions on the set of basis-functions. However, in order to argue something about non-conservativeness of the approach, one may assume that they are selected from a complete basis (in a Sobolov norm) (Johansen 2000).

\section{Performance bound computations}

\subsection{Upper and lower bounds}

Consider the performance of the system (1) or (2) with respect to a cost function

$$
\begin{array}{ll}
J(x(0))=\sum_{t=0}^{\infty} w(x(t)) & \text { D-T } \\
J(x(0))=\int_{0}^{\infty} w(x(t)) d t & \text { C-T }
\end{array}
$$


where $w(x) \geqslant 0$ is the penalty associcated with the state $x \in X$, with $w(0)=0$. This means that if the trajectory $x(t)$ converges to the origin from some initial state $x(0)$ with a sufficiently high rate, the accumulated cost $J(x(0))$ is finite and upper and lower bounded by solutions to the Hamilton-Jacobi inequalities:

$$
\begin{array}{rrr}
\bar{V}(x) \geqslant 0 & \bar{V}(f(x, \theta))-\bar{V}(x) \leqslant-w(x) & \text { UB, D-T } \\
\underline{V}(x) \geqslant 0, & \underline{V}(f(x, \theta))-\underline{V}(x) \geqslant-w(x) & \text { LB, D-T } \\
\bar{V}(x) \geqslant 0, & \frac{d \bar{V}}{d x}(x) f(x, \theta) \leqslant-w(x) & \text { UB, C-T } \\
\underline{V}(x) \geqslant 0, & \frac{d \underline{V}}{d x}(x) f(x, \theta) \geqslant-w(x) & \text { LB, C-T }
\end{array}
$$

for all $x \in X$ and $\theta \in \Theta$. LB denotes lower bound, while UB denotes upper bound. Next, define $\bar{L}, \underline{L}: X \times \Theta \rightarrow R$

$$
\begin{aligned}
\bar{L}(x, \theta) & =\bar{V}(f(x, \theta))-\bar{V}(x) & & \text { UB, D-T } \\
\underline{L}(x, \theta) & =\underline{V}(f(x, \theta))-\underline{V}(x) & & \text { LB, D-T } \\
\bar{L}(x, \theta) & =\frac{d \bar{V}}{d x}(x) f(x, \theta) & & \text { UB, C-T } \\
\underline{L}(x, \theta) & =\frac{d \underline{V}}{d x}(x) f(x, \theta) & & \text { LB, C-T }
\end{aligned}
$$

A sufficient condition for existence of a performance bound is now that there exist matrices $\bar{P}_{1}, \tilde{P}_{2}, \ldots, \bar{P}_{N}$ (upper bound) or $\underline{P}_{1}, \underline{P}_{2}, \ldots, \underline{P}$ (lower bound) such that the corresponding inequalities

$$
\begin{array}{ll}
\bar{V}(x) \geqslant 0 & \bar{L}(x, \theta) \leqslant-w(x) \quad \mathrm{UB} \\
\underline{V}(x) \geqslant 0 & \underline{L}(x, \theta) \geqslant-w(x) \quad \mathrm{LB}
\end{array}
$$

are feasible for all $x \in X$ and $\theta \in \Theta$.

Theorem 1 (Upper bound) Let X be a compact and connected set. Suppose (8) (discretetime) or (10) (continuous-time) holds for all $x \in X$ and $\theta \in \Theta$. Define $\bar{X}$ by

$$
\bar{X}=\left\{x \in X \mid \tilde{V}(x) \leqslant \inf _{\xi \in \partial X} \bar{V}(\xi)\right\}
$$

Then for all parameter trajectories $\theta(t) \in \Theta$, and $x(0) \in \bar{X} \subset X, J(x(0)) \leqslant V(x(0))$.

Proof. The result follows since $w(x) \geqslant 0$ together with (8) or (10) implies that $\bar{X}$ is an invariant set.

Theorem 2 (Lower bound) Let X be a compact and connected set. Suppose (9) (discretetime) or (11) (continuous-time) holds for all $x \in X$ and $\theta \in \Theta$. Then for all parameter trajectories $\theta(t) \in \Theta$ and state trajectories with initial conditions $x(0)$ such that $x(t) \in X$ for all $t, \underline{V}(x(0)) \leqslant J(x(0))$.

For initial conditions $x(0)$ when both bounds are well definied we have $\underline{V}(x(0)) \leqslant J(x(0)) \leqslant \bar{V}(x(0))$. 


\subsection{Stability}

If $w(x)=\gamma \bar{V}(x)$ for some constant $0<\gamma<1$ (discrete-time) or $\gamma>0$ (continuous time), $\bar{V}$ is a Lyapunov function that ensures exponential stability, see also Johansen (2000):

Theorem 3 (Exponential stability) Let $X$ be a compact and connected set. Suppose (8) (discrete-time) or (10) (continuous-time) holds with $w(x)=\gamma \bar{V}(x)$ and $\bar{V}(x) \geqslant c_{1}\|x\|_{2}^{2}$ for all $x \in X$ and $\theta \in \Theta$, where $0<\gamma<1$ (discrete-time) or $\gamma>0$ (continuous time), and $c_{1}>0$. Then for all parameter trajectories $\theta(t) \in \Theta$ and initial conditions $x(0) \in \bar{X} \subset X$, the equilibrium point is uniformly exponentially stable, i.e.

$$
\begin{aligned}
& \|x(t)\|_{2} \leqslant \sqrt{\frac{c_{2}}{c_{1}}}\|x(0)\|_{2}(1-\gamma)^{t / 2} \quad \text { D-T } \\
& \|x(t)\|_{2} \leqslant \sqrt{\frac{c_{2}}{c_{1}}}\|x(0)\|_{2} e^{-\gamma^{t} / 2} \quad \text { C-T }
\end{aligned}
$$

where $c_{2}=\max _{i} \bar{\sigma}\left(\bar{P}_{i}\right)$.

For time-invariant $\theta(t) \in \Theta$, if $w$ is such that the system is zero-state observarable (Lin and Byrnes 1995), existence of $\bar{V}$ ensures asymptotic stability with region of attraction $\bar{X}$.

Theorem 4 (Asymptotic stability) Let $X$ be a compact and connected set, and suppose $\theta(t) \in \Theta$ is time-invariant. Suppose (8) (discrete-time) or (10) (continuous-time) holds with $\bar{V}(x) \geqslant c_{1}\|x\|_{2}^{2}$ for all $x \in X$ and $\theta \in \Theta$, where $0<\gamma<1$ (discrete-time) or $\gamma>0$ (continuous time), and $c_{1}>0$. Moreover, assume the system is zero-state observable, i.e. for any trajectory $x(t) \in X, w(x(t))=0$ for all $t$ implies $x(t)=0$ for all $t$. Then for initial conditions $x(0) \in \bar{X} \subset X$, the equilibrium point is asymptoticially stable.

Proof. Consider the continuous-time case. Note that $\bar{V}(x) \geqslant c_{1}\|x\|_{2}^{2}$ is radially unbounded, and stability follows from

$$
\frac{d \bar{V}}{d t}(t) \leqslant-w(x(t)) \leqslant 0
$$

Let $S=\{x \in \bar{X} \mid w(x)=0\}$. Zero-state observability implies that the only solution that stays in $S$ is $x(t)=0$ for all $t$. Thus, by LaSalle's invariance principle, e.g. Khalil (1992), $x(t) \rightarrow 0$ as $t \rightarrow \infty$. The discrete-time case is similar.

\subsection{Linear parameterization}

Notice that $\bar{L}$ is in general a non-linear function of $x$ and $\theta$, but a linear function of the parameters of $V$ (the elements of the matrices $\bar{P}_{1}, \bar{P}_{2}, \ldots, \bar{P}_{N}$ ). In other words, it can be represented in the form $\bar{L}(x, \theta)=\bar{p}^{T} \bar{l}(x, \theta)$ where the function $\bar{l}: X \times \Theta \rightarrow R^{m}$ does not depend on the parameter vector $\bar{p} \in R^{m}$ defined by $\bar{p}=\left(\bar{P}_{1}^{1,1}\right.$, $\left.\bar{P}_{1}^{1,2}, \ldots, \bar{P}_{1}^{1, n}, \bar{P}_{1}^{2,1}, \bar{P}_{1}^{2,2}, \ldots, \bar{P}_{N}^{n, n}\right)^{T}$ and $\bar{P}_{i}^{j, k}$ is the $(j, k)$-element of the $\bar{P}_{i}$ matrix. This parameter vector has $m=N n^{2}$ elements, and the function $\bar{l}$ can be derived from (3) and (4). Furthermore, $V$ has a similar linear parametric representation $V(x)=p^{T} v(x)$ where the function $v: X \rightarrow R^{m}$ can be derived easily from (3) and (4). Hence, the condition (16)-(17) can be written as linear inequalities in the parameters $p$ : 


$$
\begin{array}{lll}
\bar{p}^{T} \underline{v}(x) \geqslant 0 & \bar{p}^{T} \underline{l}(x, \theta) \leqslant-w(x) & \text { UB } \\
\underline{p}^{T} \underline{v}(x) \geqslant 0 & \underline{p}^{T} \underline{l}(x, \theta) \geqslant-w(x) & \text { LB }
\end{array}
$$

for all $x \in X$ and $\theta \in \Theta$, where the functions and parameters involved in the lower bound are defined in an analogous manner. The constraints (22) or (23) are state- and parameter-dependent which implies that there is an infinite number of them. This leads to a so-called semi-infinite programming problem, e.g. Tanaka et al. (1988) or Polak (1997). In section 4.3, finite discretizations of the state and parameter spaces are introduced in order to reduce this infinite number of linear inequalities to a finite number of linear inequalities at the cost of an approximation. The effect of this approximation is analysed in section 4 and related to some characteristic parameters of the system and performance bound parameterization.

\subsection{Convex objective functions and constraints}

The linear inequalities (22) or (23) characterize a convex subset (a polyhedron) of the parameter space $R^{m}$ of $\bar{V}$ or $\underline{V}$. Assuming this set is non-empty (a performance bound exists), then even for very simple parameterizations of $\mathscr{V}_{N}$ (i.e. small $N$ ) there will exist an infinite number of performance bounds. Additional objectives may thus be specified in order to find a performance bound with some desirable properties, for example a simplest bound, least conservative bound or largest region of definition or attraction. This can be acheived within this framework by specifying a convex objective function that should be minimized subject to the constraints (22) or (23). Below, we will formulate linear and quadratic objective functions corresponding to these objectives. They can be selected individually or combined in a multi-objective optimization when appropriate.

3.4.1. Simple function objective The objective of finding the simplest possible function characterizing the performance bound can be formulated as follows. With the selected parameterization $\mathscr{V}_{N}$, it is natural to think of the set of quadratic functions as the simplest possible ones, corresponding to a constant function $P$, i.e. $P_{1}=P_{2}=\ldots=P_{N}$. Hence, a natural objective would be to seek matrices $P_{1}, P_{2}, \ldots, P_{N}$ that are as similar as possible. Mathematically, this is captured by the convex quadratic objective

$$
\tilde{\phi}(p)=\sum_{i=1}^{N} \sum_{l=1}^{N} \sum_{j=1}^{n} \sum_{k=1}^{n}\left(P_{i}^{j, k}-P_{l}^{j, k}\right)^{2} W_{i, l}
$$

where $W_{i, l}>0$ is some weight that in the simplest case is equal to one for all $(i, l)$, but may in general be tuned to reflect the topology of the basis-functions.

3.4.2. Minimal upper bound and maximal lower bound The average value of the upper performance bound $\bar{V}$ over $X \times \Theta$ is minimized by minimizing

$$
\bar{\phi}(\bar{p})=\int_{X \times \Theta} \bar{L}(x, \theta) s(x) d x d \theta
$$

i.e. the linear objective $\bar{\phi}(\bar{p})=\bar{p}^{T} \bar{c}$ where $\bar{c}=\int_{X \times \Theta}(\bar{l}(x, \theta)+w(x)) s(x) d x d \theta$ and $s(x) \geqslant 0$ is some weighting function, for example $s(x)=1 /\left(\varepsilon+\|x\|_{2}^{2}\right)$ where $\varepsilon>0$ is a small parameter. This also corresponds to minimizing the average decay rate when $\bar{V}$ is a 
Lyapunov function. Maximization of the lower bound in achieved by changing the sign of (25), i.e. minimizing the linear function

$$
\underline{\phi}(\underline{p})=-\int_{X \times \Theta} \underline{L}(x, \theta) s(x) d x d \theta
$$

3.4.3. Region of definition objective and constraints Suppose we impose the following linear constraints

$$
\begin{aligned}
p^{T} v(x) \geqslant q & \text { for all } x \in X-X^{b} \\
p^{T} v(x) \leqslant q & \text { for all } x \in X^{a} \\
q \geqslant 0 &
\end{aligned}
$$

where $X^{a} \subset X^{b} \subset X$. The motivation is that $\mathrm{V}$ is enforced to have a level curve $V(x)=q$ in $X^{b}-X^{a}$ which means that the performance bound (or Lyapunov function) is valid for all initial conditions $x(0) \in X^{a}$. The regions $X^{a}$ and $X^{b}$ can be defined freely. The region of definition can be maximized by letting $X-X^{b}$ and $X^{b}-X^{a}$ become small. If $X^{a}$ and $X^{b}$ have simple geometries such as balls or hyper-rectangles, this can be implemented easily by a line search for the maximum size of $X^{a}$ and $X^{b}$ when $X$ is kept fixed.

3.4.4. Average decay rate objective and constraints The objective of determining the Lyapunov function with the least conservative bound on the average decay rate can be formulated as maximization of $\gamma>0$, which can be implemented as a simple line search subject to feasibility of the constraints (22) or (23). Specification of a fixed $\gamma$ is similar to a constraint on the acceptable minimum decay rate predicted by the Lyapunov function.

\section{Discretization}

\subsection{Finite linear inequalities}

The state- and parameter-dependent linear inequalities (22) or (23) define an infinite number of linear inequalities in the finite number of parameters $p$. A finite number of linear inequalities results from discretization of the compact sets $X$ and $\Theta$ by defining finite sets $X_{d}^{0}$ and $\Theta_{d}$ containing points where the following constraints on the parameters $p$ are imposed for some $\alpha>1$ and $c_{1} \geqslant 0$

$$
\begin{array}{lll}
p^{T} v(x) \geqslant \alpha c_{1}\|x\|_{2}^{2} & p^{T} l(x, \theta) \leqslant-\alpha w(x) & \text { UB } \\
p^{T} v(x) \geqslant \alpha c_{1}\|x\|_{2}^{2} & p^{T} l(x, \theta) \geqslant-\alpha w(x) & \text { LB }
\end{array}
$$

for all $(x, \theta) \in X_{d}^{0} \times \Theta_{d}$. These inequalities are stacked in matrices as follows

$$
\begin{array}{llll}
\tilde{V} p \geqslant \tilde{c} & \text { and } & \tilde{L} p \leqslant-\alpha \tilde{W} & \text { UB } \\
\tilde{V} p \geqslant \tilde{c} & \text { and } & \tilde{L} p \geqslant-\alpha \tilde{W} & \text { LB }
\end{array}
$$

- where the rows of $\tilde{V}$ corresponds to $v^{T}(x)$ for each $x \in X_{d}^{0}$, the elements of $\tilde{c}$ are $c_{1}\|x\|_{2}^{2}$ for each $x \in X_{d}^{0}$, the rows of $L$ corresponds to $l^{T}(x, \theta)$ for each $(x, \theta) \in X_{d}^{0} \times \Theta_{d}$, and the rows of $\tilde{W}$ corresponds to $w(x)$ for each $(x, \theta) \in X_{d}^{0} \times \Theta_{d}$. Additional linear 
constraints may be due to other objectives, such as a required region of definition as discussed in section 3.4. Hence, (29) or (30) define a finite number of linear constraints in a finite number of variables, which are computationally feasible using convex linear or quadratic programming.

\subsection{Computational Procedure}

- Input data: The system function $f$, performance objective function $w$, a compact and connected set of states $X$ that contains the origin as an interior equilibrium point, and a compact parameter set $\Theta$.

- Step 1: Select a set of basis functions $\rho_{1}, \rho_{2}, \ldots, \rho_{N}$ for $\mathscr{V}_{N}$.

- Step 2: Select finite sets $X_{d} \subset X$ and $\Theta_{d} \subset \Theta$ and possibly a minimum region of definition $X^{a} \subset X^{b} \subset X$.

- Step 3: Solve the convex optimization problem of determining a feasible (or maximum) $\alpha \geqslant \alpha_{0}>1$ while minimizing one of the linear or quadratic objectives described in section 3.4 subject to the linear constraints (29) or (30) with respect to $\bar{p}$ or $p$ (there may be additional constraints if a minium region of definition is specified). If no solution was found, go to either Step 1 or Step 2.

- Step 4: Generate 'sufficiently dense' but finite checking sets $X_{c} \subset X$ and $\Theta_{c} \subset \Theta$.

- Step 5: If $\bar{L}(x, \theta) \leqslant-w(x)$ (upper bound) or $\underline{L}(x, \theta) \geqslant-w(x)$ (lower bound) does not hold on $X_{c} \times \Theta_{c}$ or $V(x) \geqslant 0$ (or $\underline{V}(x) \geqslant 0$ ) does not hold on $X_{c}$, go to either Step 1 or Step 2.

- Output data: If the procedure terminates, a performance bound has been found.

\subsection{Discretization theory}

Important information about the required granularity of design and checking points in the state and parameter spaces can be determined by analyzing the complexity of $f$ over different regions in the state subset $X$. The idea is that if $f$ is a highly nonlinear function in some regions of the state or parameter spaces, a useful heuristic may be to allow large variations in $P$ in these regions, and to increase the density of design and checking points in these regions. Define the checking set granularity function $\varepsilon$ : $X \times \Theta \rightarrow R$

$$
\varepsilon(x, \theta)=\inf _{(\xi, \zeta) \in X_{c} \times \boldsymbol{\theta}_{c}}\|(x, \theta)-(\xi, \zeta)\|_{2}
$$

The usefulness of the above mentioned heuristic can be seen theoretically by assuming $w, f, \rho_{i}$ and $d \rho_{i} / d x$ to be bounded and locally Lipschitz functions in the sense that for every $\left(x_{1}, \theta_{1}\right),\left(x_{2}, \theta_{2}\right) \in B_{\varepsilon(x, \theta)}((x, \theta))$ there exist bounded functions $L_{w}: X \rightarrow R, L_{f}$ : $X \times \Theta \rightarrow R, L_{\rho}: X \rightarrow R$ and $L_{\rho^{\prime}}: X \rightarrow R$ that satisfy

$$
\begin{gathered}
\left|w\left(x_{1}\right)-w\left(x_{2}\right)\right| \leqslant L_{w}(x)\left\|x_{1}-x_{2}\right\|_{2} \\
\left\|f\left(x_{1}, \theta_{1}\right)-f\left(x_{2}, \theta_{2}\right)\right\|_{2} \leqslant L_{f}(x, \theta)\left\|\left(x_{1}, \theta_{1}\right)-\left(x_{2}, \theta_{2}\right)\right\|_{2} \\
\left|\rho_{i}\left(x_{1}\right)-\rho_{i}\left(x_{2}\right)\right| \leqslant L_{\rho}(x)\left\|x_{1}-x_{2}\right\|_{2} \\
\left\|\frac{d \rho_{i}}{d x}\left(x_{1}\right)-\frac{d \rho_{i}}{d x}\left(x_{2}\right)\right\|_{2} \leqslant L_{\rho^{\prime}}(x)\left\|x_{1}-x_{2}\right\|_{2}
\end{gathered}
$$


and define

$$
\begin{aligned}
& K_{1}(x, \theta)=\sup _{(\xi, \zeta) \in B_{\varepsilon(x, \theta)}((x, \theta))}\|f(\xi, \zeta)\|_{2}, \quad K_{2}(x)=\sup _{\xi \in B_{\varepsilon(x)}(x)}\left\|\frac{d \rho_{i}}{d x}(\xi)\right\|_{2}, \\
& \tilde{P}=\max _{i} \bar{\sigma}\left(P_{i}\right), \quad \text { and } \quad \tilde{X}^{0}=\sup _{x, \xi \in X}\|x-\xi\|_{2} .
\end{aligned}
$$

Theorem 5 Suppose $X$ and $\Theta$ are compact sets, $w, f, \rho_{i}$ and $d \rho_{i} / d x$ are bounded and locally Lipschitz functions. Assume there exists an $\alpha>1$ such that for all $(\xi, \zeta) \in X_{c} \times \Theta_{c}$

$$
\begin{array}{ll}
\bar{L}(\xi, \zeta) \leqslant-\alpha w(\xi) & U B \\
\underline{L}(\xi, \zeta) \geqslant-\alpha w(\xi) & L B
\end{array}
$$

and the checking grid granularity $\varepsilon(x, \theta)$ is so fine that

$$
\varepsilon(x, \theta) \leqslant(\alpha-1) \frac{w(x)}{Q(x, \theta)}
$$

where in the continuous-time case

$$
\begin{aligned}
Q(x, \theta) & =\alpha L_{w}(x)+\tilde{X}^{0} \tilde{P}\left(2+N K_{2}(x) \tilde{X}^{0}\right) L_{f}(x, \theta) \\
& +K_{1}(x, \theta) \tilde{P}\left(N \tilde{X}^{0}\left(L_{\rho^{\prime}}(x) \tilde{X}^{0}+2 K_{2}(x)+2 L_{\rho}(x)\right)+2\right)
\end{aligned}
$$

and in the discrete-time case

$$
Q(x, \theta)=\alpha L_{w}(x)+2\left(\tilde{X}^{0}\right)^{2} \tilde{P}\left(\tilde{X}^{0}+K_{1}(x, \theta) L_{f}(x, \theta)\right)
$$

Then for all $x \in X$ and $\theta \in \Theta$

$$
\begin{aligned}
& L(x, \theta) \leqslant-w(x) \quad U B \\
& \underline{L}(x, \theta) \geqslant-w(x) \quad L B
\end{aligned}
$$

Proof. The proof is similar to Johansen (2000). Consider first the upper bound:

$$
\begin{aligned}
L(x, \theta) & =L(\xi, \zeta)+(L(x, \theta)-L(\xi, \zeta)) \\
& \leqslant-\alpha w(\xi)+(L(x, \theta)-L(\xi, \zeta)) \\
& =-w(x)-(\alpha-1) w(x)+\alpha(w(x)-w(\xi))+(L(x, \theta)-L(\xi, \zeta)) \\
& \leqslant-w(x)-(\alpha-1) w(x)+Q(x, \theta) \varepsilon(x, \theta)
\end{aligned}
$$

where the last inequality can be verified from the definition of $Q(x, \theta)$ using the Schwarz inequality and the Lipschitz properties. The result now follows due to (34). The lower bound can be verified in a completely analogous manner.

\section{Example}

Consider the discrete-time double integrator

$$
A=\left(\begin{array}{ll}
1 & 1 \\
0 & ,
\end{array}\right), \quad B=\left(\begin{array}{l}
0 \\
\theta
\end{array}\right)
$$


where the nominal value of the paramter $\theta=1$. The control objective is defined by the cost function

$$
\begin{gathered}
\mathscr{L}(x(0))=\sum_{t=0}^{\infty} l(x(t), u(t)) \\
l(x, u)=x_{1}^{2}+u^{2}
\end{gathered}
$$

and the constraints

$$
\begin{gathered}
-0.5 \leqslant x_{2} \leqslant 0.5 \\
-1 \leqslant u \leqslant 1
\end{gathered}
$$

If $x_{1}$ is interpreted as position, $x_{2}$ as speed and $u$ as force, the objective is to control the position under constraints on the speed and force. Using the suboptimal constrained LQR method proposed in Johansen et al. (2000), a piecewise linear static state feedback $u=g(x)$ is designed, cf. Figure 1 . The locally linear function near the origin corresponds to the linear feedback $u=K x$ of the classical unconstrained LQ controller, while the remaining linear pieces are introduced to avoid violating the constraints. The closed loop system is now

$$
x(t+1)=A x+B g(x)
$$

Note that due to continuity of the piecewise linear function $g$, the right-hand-side of (46) defines a continuous piecewise linear function that satisfy the Lipschitz property. The closed loop system performance is

$$
\begin{aligned}
J(x(0)) & =\sum_{t=0}^{\infty} w(x(t)) \\
w(x) & =l(x, g(x))=x_{1}^{2}+g^{2}(x)
\end{aligned}
$$

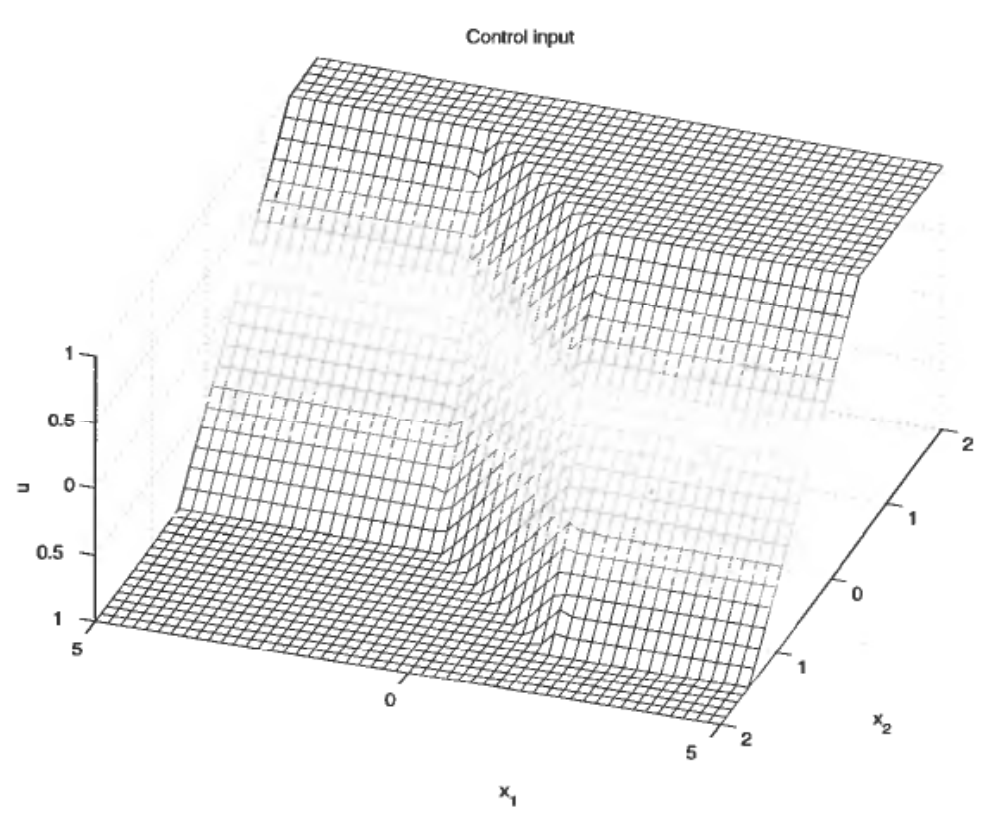

Figure 1. Suboptimal piecewise linear controller surface. 


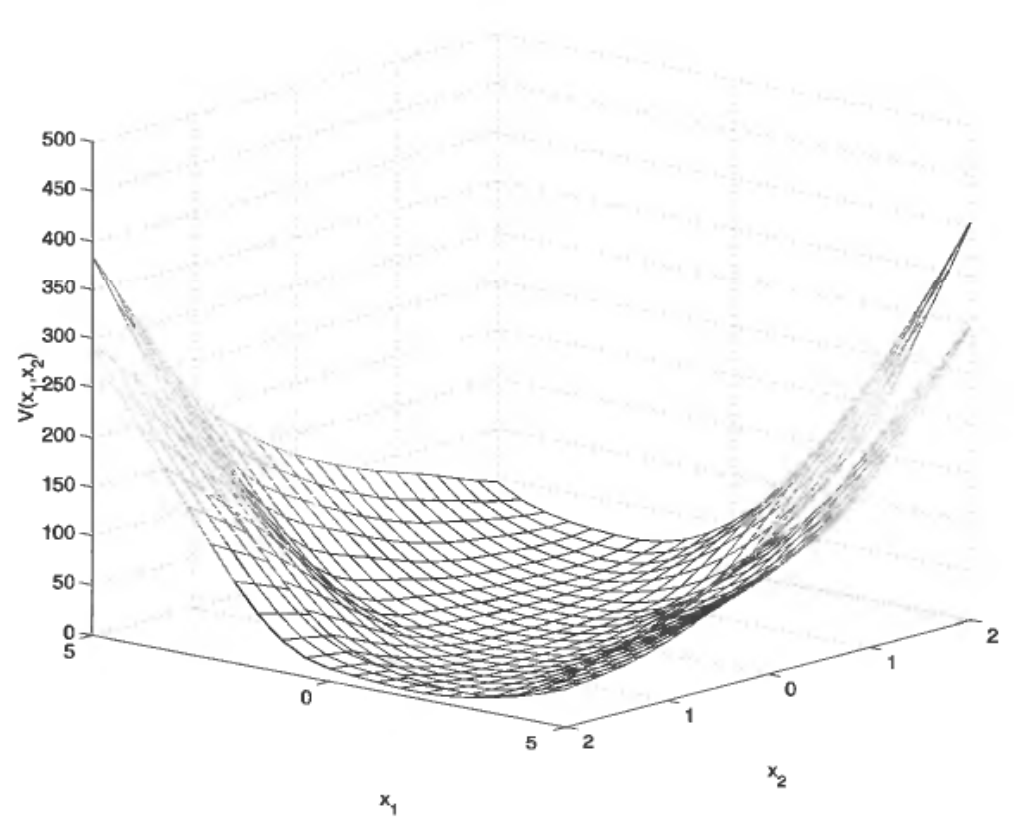

Figure 2. Upper and lower nominal performance bounds.

Assuming $\theta=1$ (nominal case), upper and lower bounds $\bar{V}$ and $\underline{V}$ on $J$ are computed using the procedure described above, and is illustrate in Figure 2. In this case the function is parameterized by 16 normalized Gaussian basis-functions (Johansen 2000), leading to 64 free parameters defining $V$ and $\bar{V} .441$ grid points are used, leading to LP/QP problems with 64 variables and between $882-323$ inequalities, which typically requires computation times of a few seconds. Results for three different initial conditions, with $\theta=1$ are given in Table 1 .

The system is zero-state observable (since $x_{1}(t)=0$ for all $t$ is possible only if $x_{2}(t)=0$ for all $\left.t\right)$. Hence, the upper bound on the performance $\vec{V}$ defines a Lyapunov function, cf. Figure 3. Note, however, that $\bar{V}$ is optimized to minimize the average upper bound rather than maximize the region of attraction. Taking $w(x)=\gamma \bar{V}(x)$ and maximizing the region of attraction and the decay rate $\gamma>0$ we achieve the alternative Lyapunov function $V(x)$ in Figure 3, lower part, with $\gamma=0.125$. It is observed that this Lyapunov function estimates a significantly larger region of attraction, as expected. Next, consider that case when $\theta(t) \in[0.75,1.25]$ is uncertain and possibly time-varying. In this case, upper and lower bounds on the performance are computed using the same parameterization and procedure as above. The performance bounds are illustrated in Figure 4. Comparing with the nominal case, Figure 2, we observe that due to the

Table 1. Comparison of actual cost with computer upper and lower bounds, with no uncertainty taken into account.

\begin{tabular}{lrrr}
\hline$x(0)$ & $\underline{V}(x(0))$ & $f(x(0))$ & \multicolumn{1}{c}{$\bar{V}(x(0))$} \\
\hline$(0.3,0.3)^{T}$ & 0.3398 & 0.9084 & 1.0110 \\
$(-2,0)^{T}$ & 10.3974 & 11.8521 & 16.2764 \\
$(3,1)^{T}$ & 71.4624 & 77.3501 & 89.5896 \\
\hline
\end{tabular}



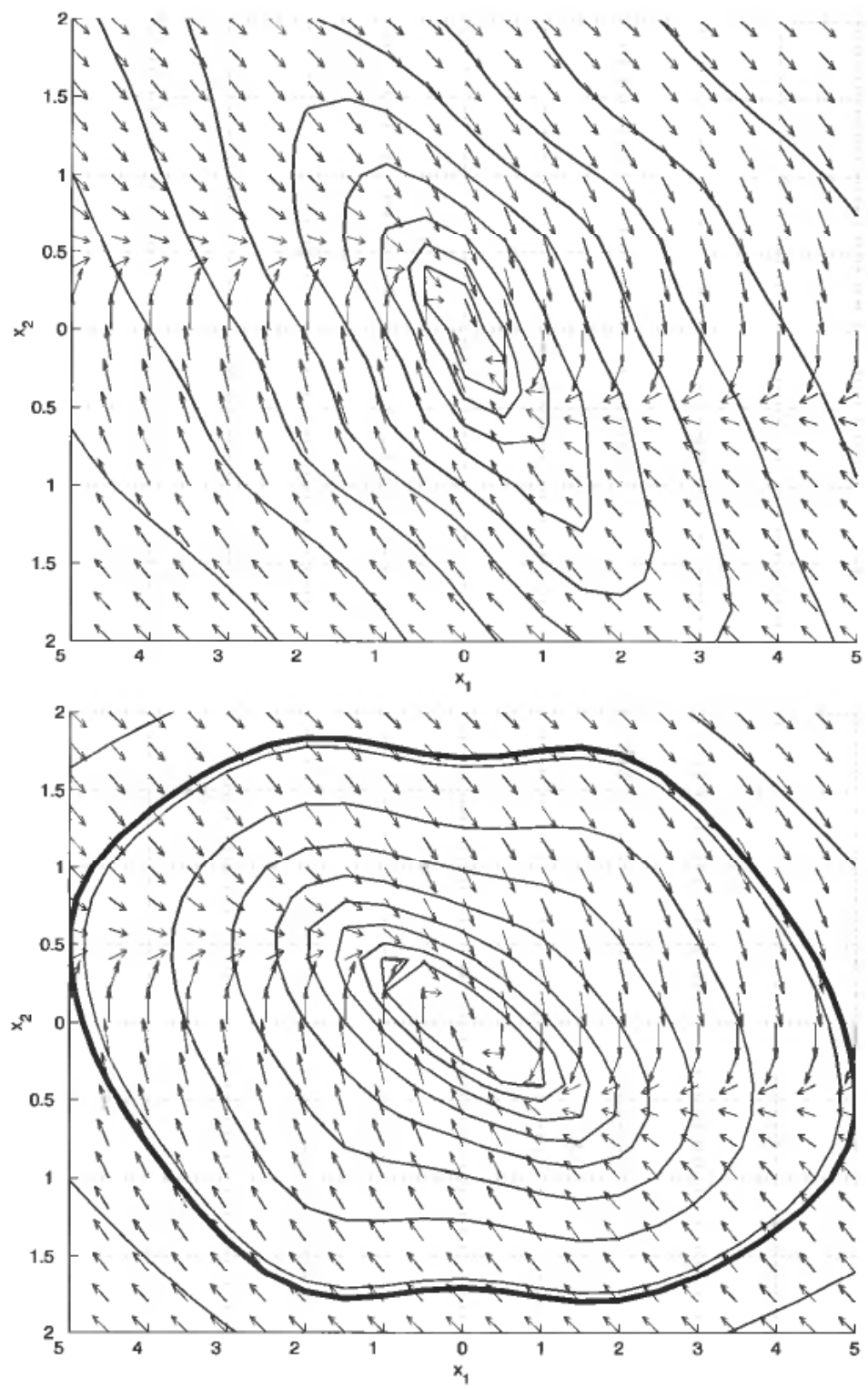

Figure 3. Upper; Level curves for the Lyapunov function derived from upper nominal performance bound. Lower: Level curves for the Lyapunov function computed by maximizing regionu of attraction for the nominal system. The arrows indicate the flow of the system, i.e. the direction of the vector $x(t+1)-x(t)$. Note that the vectors are normalized to have the same length.

uncertainty, the gap between the upper and lower bounds is significantly larger than in the nominal case. A Lyapunov function computed by explicitly maximizing the region of attraction subject to the uncertain parameter $\theta$ is given in Figure 5 .

\section{Concluding Remarks}

A computational approach to analysis of performance and stability of a general class of nonlinear systems is considered. It is shown that useful smooth non-quadratic 


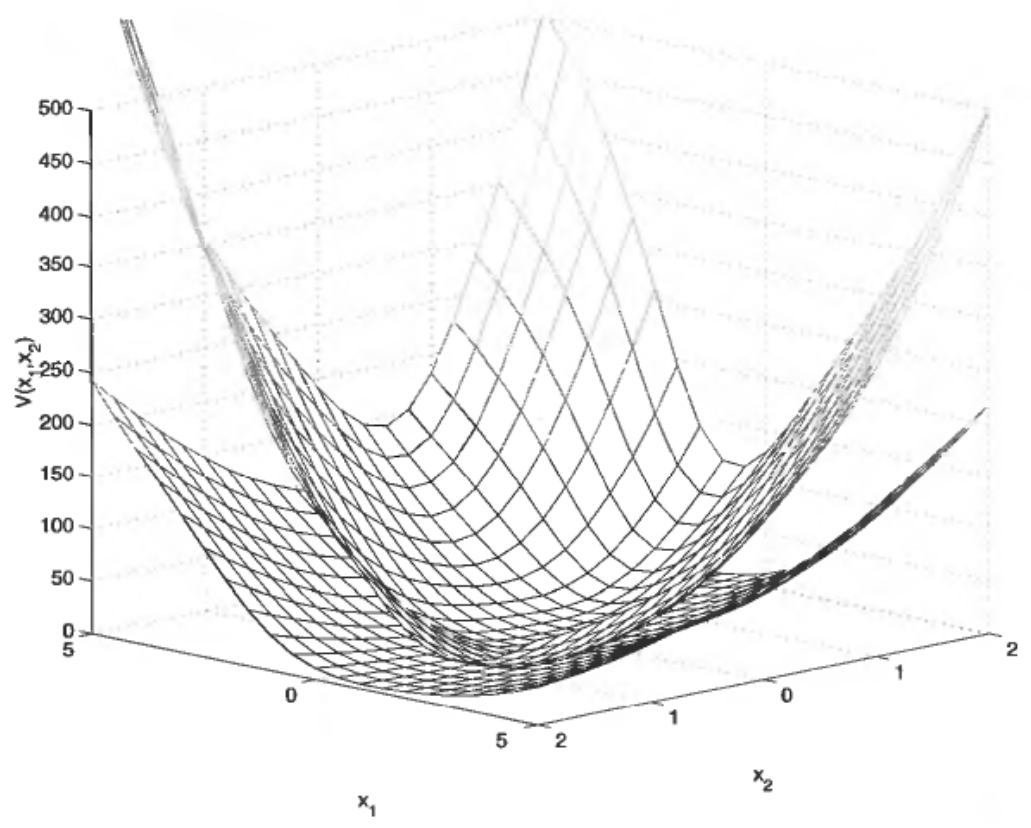

Figure 4. Upper and lower performance bounds (uncertain system).

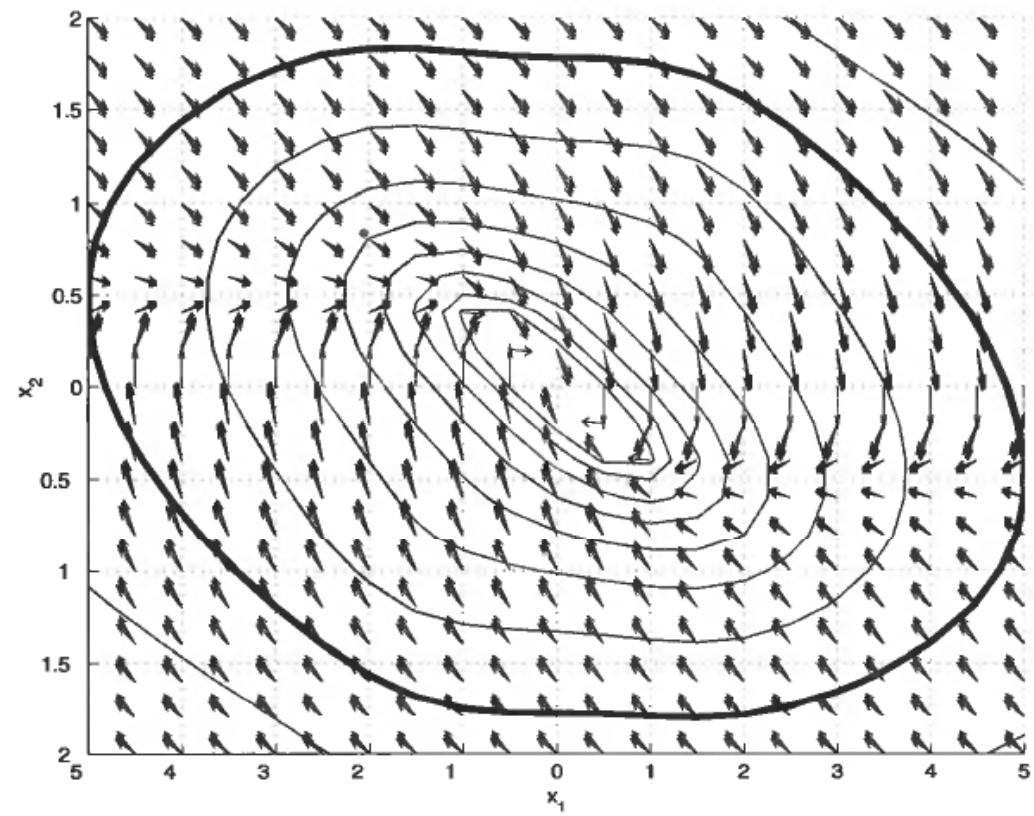

Figure 5. Level curves for the Lyapunov function computed by maximizing region of attraction (uncertain system). The arrows indicate the flow of the system, i.e. the direction of the vector $x(t+1)-x(t)$, for the nominal and extreme values of the parameters $\theta \in\{0.75,1,1.25\}$. Note that the vectors are normalized to have the same length. 
performance bounds and Lyapunov functions can be computed using semi-infinite linear or quadratic programming. The methods studied are inherently prone to the curse of dimensionality and will certainly not be feasible for large-scale problems. However, there are many complex nonlinear dynamical systems with a total of 2-4 states and uncertain parameters where the methods can be readily applied. In order to extend the applicability to problems with perhaps up to 5-7 states and parameters it is of interest to investigate numerical methods to be used to efficiently solve the LP/ QP. In particular, multi-resolution methods seems to be a promising alternative. Moreover, in order to reduce the computational complexity of the optimization problem it would also be useful to study methods for automatically generating non-uniform grids of discretization points.

\section{REFERENCES}

Johansen, T. A. (2000). Computation of Lyapunov Functions for Smooth Nonlinear Systems Using Convex Optimization. Automatica, Vol. 36.

Rantzer, A. (1999). Dynamic Programming Via Convex Optimization. In: Preprints IFAC World Congress, Beijing, Vol. D, pp. 491-496.

Johansson, M. and Rantzer, A. (1998). Computation of Piecewise Quadratic Lyapunov Functions for Hybrid Systems. IEEE Trans. Automatic Control, Vol. 43, pp. 555-559.

Rantzer, A. and Johansson, M. (2000). Piecewise Linear Quadratic Optimal Control. IEEE Trans. Automatic Control, Vol. 45, pp. 629-637.

Petterson, S. and Lennartson, B. (1997). Exponential Stability Analysis of Nonlinear Systems Using LMIs. In: Proc. IEEE Conf. Decision and Control, San Diego.

JoHANSEN, T. A. (1999). Characterization of Lyapunov Functions for Smooth Nonlinear Systems Using LMIs. In: Preprints IFAC World Congress, Beïing, Vol. 3, pp. 387-392.

OнtA, Y. and ONISHI, M. (1999). Stability Analysis by Using Piecewise Linear Lyapunov Functions. In: Preprints IFAC World Congress, Beijing, Vol. D, pp. 515-520.

Julian, P., Guivant, J. and Desages, A. (1999). A Parameterization of Piecewise Linear Lyapunov Functions via Linear Programming. Int. J. Control, Vol. 72, pp. 702-715.

Branicky, M. D. (1995). Studies in Hybrid Systems: Modeling, Analysis and Control. PhD Thesis, MIT, Laboratory for Information and Decision Systems.

Hedlund, S. and Rantzer, A. (1999). Optimal Control of Hybrid Systems. In: Proc. IEEE Conference on Decision and Control, Phoenix, AZ.

Lin, W. and ByrneS, C. I. (1995). Zero-state Observability and Stability of Discrete-time Nonlinear Systems. Automatica, Vol. 31, pp. 269-274.

KHALIL, H. K. (1992). Nonlinear Systems. Macmillan, New York.

TANaKa, Y., Fukushima, M. and Ibaraki, T. (1988). A Comparative Study of Several Semiinfinite Nonlinear Programming Algorithms. European Journal of Operational Research, Vol. 36, pp. 92-100.

PolaK, E. (1997). Optimization: Algorithms and Consistent Approximation. Springer, New York.

Johansen, T. A., Petersen, I. and SlupPHaug, O. (2000). Explicit Suboptimal Linear Quadratic Regulation with Input and State Constraints. Proc. IEEE CDC, Sydney. 